\title{
Orientación religiosa y solución de problemas*
}

\author{
Yefren Díaz López \\ Adelia de Miguel Negredo***
}

Recibido: 20 de agosto de 2015 - Aprobado: 12 de diciembre de 2015

\section{Besumen}

Este artículo presenta los resultados de los aportes de la orientación religiosa a la solución de problemas en personas de confesionalidad cristiana católica y protestante pentecostal de la ciudad de Bogotá, Colombia, desde un enfoque cognitivo conductual en psicología de la religión, y un diseño factorial 2×3×4, donde los factores confesionalidad cristiana, locus de control y orientación religiosa se relacionan con las variables criterio propias de la dimensión constructiva y disfuncional de solución de problemas sociales de D'Zurilla y Goldfried. La muestra fue equilibrada: 45 personas de confesionalidad católica y 45 protestantes pentecostales, a quienes se les aplicó la escala de orientación religiosa de Allport y Ross, la escala de locus de control de Rotter y el inventario de solución de problemas sociales revisado. Se encontró que las personas sin entrenamiento previo en solución de problemas sociales, con mayores puntuaciones en la dimensión constructiva y menores en la dimensión disfuncional tienden a la orientación religiosa internalizada independiente de su confesionalidad cristiana; las personas con orientación religiosa internalizada presentan correlaciones significativas y positivas con la dimensión constructiva, y correlaciones significativas y negativas con la puntuación de la orientación religiosa externalizada; y las personas con orientación religiosa internalizada y locus de control interno tienden a obtener altas puntuaciones en la dimensión constructiva, mientras que las personas con orientación religiosa externalizada y locus de control externo tienden a puntuar más en la dimensión disfuncional.

Palabras clave: orientación religiosa, experiencia religiosa, psicología, religión, solución de problemas.

Artículo de investigación. Este trabajo presenta algunos resultados de la tesis doctoral: Aportes de la experiencia religiosa a la intervención psicológica en solución de problemas online: una aproximación interdisciplinar. Facultad de Ciencias de la Salud, Sección de Psicología, Departamento de Psicología Clínica, Psicobiología y Metodología. Universidad de La Laguna, Santa Cruz de Tenerife, España. DOI: http://dx.doi.org/10.15332/s0120-8454.2016.0089.05

* Doctor en Psicología de la Universidad de la Laguna, Santa Cruz de Tenerife, España. Magíster en Desarrollo Educativo y Social de la Fundación Centro Internacional de Educación y Desarrollo Humano (Cinde), Universidad Pedagógica Nacional (UPN), Bogotá, Colombia. Psicólogo de la Fundación Universitaria Konrad Lorenz, Bogotá, Colombia. Licenciado en Teología de la Pontificia Universidad Javeriana, Bogotá Colombia. Docente del Centro de Formación Teológica y coordinador académico de la Licenciatura en Ciencias Religiosas de la Facultad de Teología de la Pontificia Universidad Javeriana, Bogotá, Colombia. Dirección postal: Carrera 5. \# 39-00, edificio Pedro Arrupe, S. J., Facultad de Teología, Bogotá, Colombia. Correo electrónico: yefren.diaz@javeriana.edu.co.

... Doctora en Psicología. Licenciada en Filosofía y Ciencias de la Escuela Catedrática. Coordinadora del grupo de investigación Personalidad (Personalidad, identidad de género y orientación afectivo-sexual) y Envejecimiento de Universidad de La Laguna. Dirección postal: Pabellón de Gobierno, C/ Padre Herrera s/n. 38200, apartado postal 456, La Laguna, España. Correo electrónico: admiguel@ull.es. 


\title{
Religious guidance and problem solving*
}

\author{
Yefren Díaz López \\ Adelia de Miguel Negredo**
}

A bstract

This article presents the results of the contributions of the religious orientation to solving problems in Catholic-Christian and Protestant-Pentecostal people in Bogotá, Colombia, from a cognitive-behavioral approach in psychology of religion, and $2 \times 3 \times 4$ factorial design where the Christian confessional, locus of control and religious orientation factors are related to the constructive and dysfunctional dimension variables of solving social problems of Goldfried D'Zurilla. The sample was balanced: the religious orientation scale of Allport and Ross, the Rotter locus of control scale and revised inventory of solving social problems were applied to 45 Catholic people and 45 Pentecostal Protestants. We found that people without previous training in solving social problems with higher scores in the constructive and smaller dimension in the dysfunctional dimension tend to an internalized religious orientation apart from their Christian denominational; people with internalized religious orientation have significant and positive correlations with the constructive dimension and significant and negative correlations with externalized religious orientation score; and people with internalized religious orientation and internal locus of control tend to get high scores on the constructive dimension, while people with externalized religious orientation and external locus control tend to score more in the dysfunctional dimension.

Keywords: religious experience, psychology, religion.

Research paper. This paper presents some results of the doctoral thesis: Contributions of the religious experience to psychological intervention in online problem solving: an interdisciplinary approach. Faculty of Health Sciences, Section of Psychology, Department of Clinical Psychology, Psychobiology and Methodology. Universidad de La Laguna, Santa Cruz de Tenerife, Spain. DOI: http://dx.doi.org/10.15332/s0120-8454.2016.0089.05

* Doctor in Psychology from Universidad de la Laguna, Santa Cruz de Tenerife, Spain. Master in Educational and Social Development from Fundacion Centro Internacional de Educación y Desarrollo Humano (Cinde), Universidad Pedagogica Nacional (UPN), Bogota, Colombia. Psychologist from Fundacion Universitaria Konrad Lorenz, Bogota, Colombia. Bachelor of Theology from Pontificia Universidad Javeriana, Bogota, Colombia. Professor of the Theological Training Center and academic coordinator of the Degree in Religious Studies of the Faculty of Theology at Pontificia Universidad Javeriana, Bogota, Colombia. Address: Carrera 5a \# 39-00, building Pedro Arrupe, S.J. Faculty of Theology, Bogota, Colombia. Email: yefren.diaz@javeriana.edu.co.

.*. PhD in Psychology. Degree in Philosophy and Sciences of Escuela Catedratica. Coordinator of the Personality (Personality, gender identity and sexual- affective orientation) and Aging research group from Universidad de La Laguna. Address: Pabellon de Gobierno, C/ Padre Herrera s/n. 38200, PO Box 456, La Laguna, Spain. Email: admiguel@ull.es. 


\title{
Orientation religieuse et solution de problèmes*
}

\author{
Yefren Díaz López ${ }^{* *}$ \\ Adelia de Miguel Negredo**
}

\section{Résumé}

Cet article présente les résultats des apports de l'orientation religieuse à la solution de problèmes chez des personnes de confession chrétienne-catholique et protestante- pentecôtiste de la ville de Bogota, Colombie, à partir d'un point de vue cognitif et comportemental dans la psychologie de la religion, et un schéma factoriel $2 \times 3 \times 4$, où les facteurs de confession chrétienne, locus de contrôle et orientation religieuse se lient avec les variables critères propres de la dimension constructive et dysfonctionnelle de la solution de problèmes sociaux de D'Zurilla et Goldfried. L'échantillon fût équilibré: 45 personnes de confession catholique et 45 protestantes-pentecôtistes, à qui l'on a appliqué l'échelle d'orientation religieuse d'Allport et Ross, l'échelle de locus de contrôle de Rotter et l'inventaire de solution de problèmes sociaux révisé. On a trouvé que les personnes sans un entraînement antérieur dans la solution de problèmes sociaux, avec de meilleurs résultats dans la dimension constructive, et moins bons dans la dimension dysfonctionnelle tendent à une orientation religieuse intériorisée indépendante de leur confession chrétienne; les personnes avec une orientation religieuse intériorisée présentent des corrélations significatives et positives avec la dimension constructive, et des corrélations significatives et négatives avec le classement de l'orientation religieuse externalisée; et les personnes avec une orientation religieuse intériorisée et locus de contrôle interne tendent à avoir plus de points dans la dimension constructive, tandis que les personnes avec une orientation religieuse externalisée et locus de contrôle externe tendent à avoir plus de points dans la dimension dysfonctionnelle.

Mots clés: orientation religieuse, expérience religieuse, psychologie, religion, solution de problèmes.

Article de recherche. Ce travail présente quelques résultats de la thèse doctorale: Aportes de l'experiencia religiosa a la intervención psicológica en solución de problemas online: una aproximación interdisciplinar, Apports de l'expérience religieuse à l'intervention psychologique dans la solution de problèmes online: une approche interdisciplinaire. Faculté de Sciences de la Santé, Section Psychologie, Département de Psychologie Clinique, Psychobiologie et Méthodologie. Université de La Laguna, Santa Cruz de Tenerife, Espagne. DOI: http://dx.doi.org/10.15332/s0120-8454.2016.0089.05

** Docteur en Psychologie de I'Université de la Laguna, Santa Cruz de Tenerife, Espagne. Magister en Développement Éducatif et Social de la Fondation Centre International d’Éducation et Développement Humain (Cinde), Université Pédagogique Nationale (UPN), Bogota, Colombie. Psychologue de la Fondation Universitaire Konrad Lorenz, Bogota, Colombie. Licencié en Théologie de I'Université Javeriana, Bogota, Colombie. Professeur du Centre de Formation Théologique et coordinateur académique du cursus en Sciences Religieuses de la Faculté de Théologie de I'Université Javeriana, Bogota, Colombie. Adresse postale: Carrera 5. \# 39-00, edificio Pedro Arrupe, S. J., Faculté de Théologie, Bogota, Colombie. E-mail: yefren.diaz@javeriana.edu.co.

*** Docteur en Psychologie. Licenciée en Philosophie et Sciences de I'École Catedrática. Coordinatrice du groupe de recherche Personnalité (Personnalité, identité de genre et orientation afectivo-sexuelle) et Vieillissement de I'Université de La Laguna. Adresse postale: Pabellón de Gobierno, C/ Padre Herrera s/n. 38200, apartado postal 456, La Laguna, Espagne. E-mail: admiguel@ull.es. 


\section{Introducción}

Este artículo presenta los resultados de los aportes de la orientación religiosa a la solución de problemas en personas de confesionalidad cristiana católica y protestante pentecostal de la ciudad de Bogotá, Colombia, desde un enfoque cognitivo-conductual de la psicología de la religión, cuyo objeto de estudio es la experiencia religiosa del hombre que se confiesa creyente, con la intencionalidad de "llegar a un entendimiento de las bases psicológicas de la creencia religiosa, la experiencia y el comportamiento" (Emmons y Paloutzian, 2003, p. 378).

El camino racional que sigue la ciencia no se opone a la experiencia religiosa fundamentada en la experiencia humana, pues, "aunque el término de la vivencia religiosa es algo trascendente, se trata sin embargo, de una experiencia humana, propia del ser humano y condicionada por su forma de ser y su contexto histórico y cultural" (Croatto, 2002, p. 37) En consecuencia, la inteligencia del creyente se esfuerza en asimilar la verdad revelada, desde la Palabra que responde a las preguntas más profundas del ser humano en general, y del creyente cristiano en particular, de "la forma racional y científica que es propia al entendimiento humano." (Comisión Teológica Internacional, 2012, p. 57).

Por lo tanto, en los procesos de solución de problemas sociales, cobra importancia la aproximación a la experiencia religiosa desde las implicaciones de la variable orientación religiosa, en busca de superar experiencias religiosas sesgadas por prejuicios -irracionales - . Lo anterior lleva a definir la orientación religiosa como la forma en que un individuo se aproxima a (o evita) la religión.

De aquí se sigue que la psicología de la religión deja la indagación acerca de la verdad divina a la teología, para fundamentar la medida de la orientación religiosa en los hallazgos empíricos y teóricos a partir de los cuales profundiza y analiza críticamente las realidades religiosas en las realidades humanas de los creyentes. Es el caso de Allport, quien parte en su propuesta inicial de la teoría de la religión madura e inmadura, para luego dar el paso a la teoría de la religión intrínseca y extrínseca, que es una de las variables independientes utilizadas en esta investigación.

Identificando que la orientación extrínseca está guiada por valores extrínsecos y tiene una tendencia a lo institucional, instrumental y utilitario, mientras que la religión intrínseca es dinámica, lo que implica una forma de fe guiada por la probabilidad y no por certeza, es completa - al asumir la comprensión religiosa como filosofía integral de la vida -, es bien integrada - al estar bien articuladas sus creencias-, y produce una moral coherente. Lo anterior lleva a que Patrick (1979) concluya, en relación con la religiosidad intrínseca de Allport, que por su "falta de contenido doctrinal y definición abierta de la religión permite su uso con prácticamente cualquier denominación cristiana, y tal vez incluso con las religiones no cristianas" (Donahue, 1985, p. 415). 
Igualmente, reconociendo la aproximación de $\operatorname{Rotter}(1975,1978)$ a la conexión conceptual entre las teorías del reforzamiento y las teorías cognitivas, se sigue en esta investigación que en el desarrollo de habilidades cognitivas y conductuales es importante considerar, a partir de las diferencias individuales, la percepción que del refuerzo pueden llegar a tener las personas, la cual, por un lado, puede ser contingente al propio comportamiento y, por el otro, ser independiente de aquel.

Lo anterior significa, en el caso de las personas con opción religiosa, que la variable locus de control hace referencia a las creencias que se tienen sobre si los resultados que experimentan dependen de sus acciones o no, lo que lleva en esta línea de investigación a evidenciar "que a más externo el locus de control, menos orientación religiosa intrínseca, y viceversa" (Syeda y Rabia, 2011, p. 71), para finalmente abordar los procesos de solución de problemas desde el enfoque cognitivo conductual de D'Zurilla y Goldfried (1971), quienes plantean modificar la conducta a partir del empleo de la teoría e investigación acumulada en solución de problemas, con el objetivo de facilitar la generalización de los cambios y habilidades aprendidas, facilitando que el consultante llegue a funcionar como su propio terapeuta.

Los procesos que caracterizan el modelo de D'Zurilla y Goldfried (1971) se agrupan alrededor de dos dimensiones. La dimensión constructiva se refiere al proceso de orientación positiva al problema y al proceso de estilo racional de solución de problemas, y la dimensión disfuncional incluye el proceso de orientación negativa al problema, el proceso de estilos impulsivo/descuidado y el proceso evitativo de solución de problemas.

El modelo predice que las puntuaciones altas de los sujetos en la orientación positiva hacia el problema facilita los resultados positivos desde el estilo racional de solución de problemas, contrario a las puntuaciones bajas de los sujetos en orientación negativa hacia el problema, que tiende a los resultados negativos desde los estilos impulsivo/descuidado y evitativo de solución de problemas.

Partiendo de la relación significativa encontrada en diferentes investigaciones, entre la orientación religiosa y el comportamiento de las personas, se aborda puntualmente la relación entre orientación religiosa y la dimensión constructiva y disfuncional de solucionar problemas, teniendo como presupuesto que la orientación religiosa puede llevar a conductas de control o autocontrol de la vida de las personas con creencias religiosas.

\section{Método}

\section{Participantes}

La muestra fue incidental y estuvo conformada por 90 participantes cristianos católicos y protestantes pentecostales de la ciudad de Bogotá (Colombia). La confesionalidad cristiana de los participantes estuvo balanceada $50 \%$ y $50 \%$. 


\section{Instrumentos}

Escala de orientación religiosa (ROs. Allport y Ross, 1967), con un alfa de Cronbach de 0,70 para la escala extrínseca y 0,73 para la escala intrínseca.

Escala de locus de control (Locus de control, Rotter, 1966), con un alfa de Cronbach de 0,65 .

Inventario de solución de problemas sociales revisado (sPsi-R. D'Zurilla et al., 1999), con un alfa de Cronbach mayor a 0,90 (D'Zurilla et al., 1999).

El diseño factorial tiene tres factores. El primer factor, confesionalidad cristiana, actúa en dos niveles: católicos y protestantes penteostales. El segundo factor, locus de control, tiene tres niveles: locus de control interno, locus de control medio y locus de control externo. Y el tercer factor, orientación religiosa, tiene cuatro niveles: orientación religiosa internalizada, orientación religiosa externalizada, orientación religiosa prorreligiosa, orientación religiosa no-religiosa. Las variables dependientes fueron las puntuaciones obtenidas en el inventario de solución de problemas en la dimensión constructiva y disfuncional.

\section{Resultados}

El análisis de datos se realizó a través del software spss, versión 2.0, teniendo en cuenta como variables independientes la orientación religiosa clasificada, como internalizada, externalizada, prorreligiosa, no-religiosa; el locus de control, con los niveles interno, medio y externo; y el grupo, siendo uno de confesionalidad católica y el otro de confesionalidad protestante pentecostal, los dos sin este entrenamiento previo en solución de problemas. Las variables dependientes fueron las puntuaciones obtenidas en el inventario de solución de problemas en la tendencia a la dimensión constructiva y la dimensión disfuncional evaluada en las muestras independientes.

Para evaluar la diferencia en las puntuaciones de las dimensiones del SPSI-R en los dos grupos, se partió de las puntuaciones obtenidas en los dos factores de la escala Ros, lo que permitió clasificar a los participantes en cuatro niveles, en función de las medianas ( 34 y 32 para orientación internalizada y externalizada, respectivamente): no religioso: puntuaciones totales menores que las medianas de muestra total en ambos factores de orientación religiosa internalizada y externalizada; prorreligioso: puntuaciones totales mayores que las medianas de puntuaciones en ambos factores de orientación religiosa internalizada y externalizada; internalizada: puntuación total en el factor de orientación religiosa internalizada mayor a la mediana y puntuación total en el factor de orientación religiosa externalizada menor a la mediana; y externalizada: puntuación total en el factor de orientación religiosa externalizada mayor a la mediana y puntuación total en el factor de orientación religiosa internalizada menor a la mediana. 
Teniendo en cuenta el anterior criterio de clasificación, se tiene el $26,7 \%$ de participantes con orientación religiosa externalizada, el 16,7\% de prorreligiosos, el $30 \%$ con orientación religiosa internalizada y el $26,7 \%$ con orientación no religiosa.

$\mathrm{Al}$ igual que en el caso de la escala Ros, la puntuación continua obtenida en el cuestionario de locus de control también se transformó en una variable dummy, con tres valores: locus de control interno: se consideran personas con tendencia al locus de control interno a aquellas con puntuaciones directas menores al percentil 33 (rango de puntuaciones 0-6); con locus de control medio, a aquellas con puntuaciones entre el percentil 33 y 67 (rango de puntuaciones 7-9); y con locus de control externo, a aquellas con puntuaciones superiores al percentil 67 (rango de puntuaciones 10-16).

A partir del anterior criterio de clasificación, los participantes con locus de control externo representan el $31,1 \%$ de la muestra, mientras que el $36,7 \%$ corresponde a participantes con locus de control interno. El locus de control medio representa al 32,2\% de participantes en las pruebas de independencia entre confesionalidad cristiana y locus de control. En la tabla 1 se observa que entre católicos y protestantes pentecostales existen proporciones diferentes en el locus de control interno y externo. La prueba Chi cuadrado $(=14,019 ; \mathrm{p}=0,001)$ confirma la dependencia entre el locus de control y la confesionalidad cristiana.

Respecto a las pruebas de independencia entre confesionalidad cristiana y orientación religiosa, se identifica que entre los participantes católicos hay una tendencia a clasificarse de acuerdo con la prueba de orientación religiosa hacia lo internalizado, seguido de la orientación pro religiosa, mientras que en los grupos de protestantes pentecostales fueron más frecuentes los casos de orientación religiosa externalizada y orientación religiosa prorreligiosa. Como se observa en la tabla 2 , la prueba Chi cuadrado $(=6,181 ; \mathrm{p}=0,087 ; \alpha=0,05)$ confirma la independencia entre la orientación religiosa y la confesionalidad (católica/protestante pentecostal).

Tabla 1. Contingencia confesionalidad cristiana con locus de control en los tres niveles

\begin{tabular}{|c|c|c|c|c|c|c|c|c|}
\hline \multirow{2}{*}{$\begin{array}{l}\text { Locus } \\
\text { de } \\
\text { control }\end{array}$} & \multicolumn{3}{|c|}{ Católico } & \multicolumn{3}{|c|}{ Protestante-p } & \multirow[b]{2}{*}{$\mathbf{N}$} & \multirow[t]{2}{*}{ Confesionalidad } \\
\hline & $\mathrm{n}$ & $\begin{array}{l}\text { Dentro de } \\
\text { religión }\end{array}$ & $\begin{array}{l}\text { Dentro de } \\
\text { Loc_ctrol_ } \\
\text { tres_niveles }\end{array}$ & $\mathrm{n}$ & $\begin{array}{l}\text { Dentro } \\
\text { de } \\
\text { religión }\end{array}$ & $\begin{array}{l}\text { Dentro de } \\
\text { locus de } \\
\text { control }\end{array}$ & & \\
\hline & & $\%$ & $\%$ & & $\%$ & $\%$ & & \\
\hline Interno & 25 & 55,6 & 75,8 & 8 & 17,8 & 24,2 & 33 & 36,7 \\
\hline Medio & 11 & 24,4 & 37,9 & 18 & 40,0 & 62,1 & 29 & 32,2 \\
\hline Externo & 9 & 20,0 & 32,1 & 19 & 42,2 & 67,9 & 28 & 31,1 \\
\hline Total & 45 & 100 & 50 & 45 & 100 & 50 & 90 & 100,0 \\
\hline
\end{tabular}

Nota: Protestante- $\mathrm{p}=$ protestante-pentecostal; $\mathrm{n}$ = número de casos del total; $\mathrm{N}=$ total de número de caso; Loc_ctrol = locus de control.

Fuente: elaboración propia. 


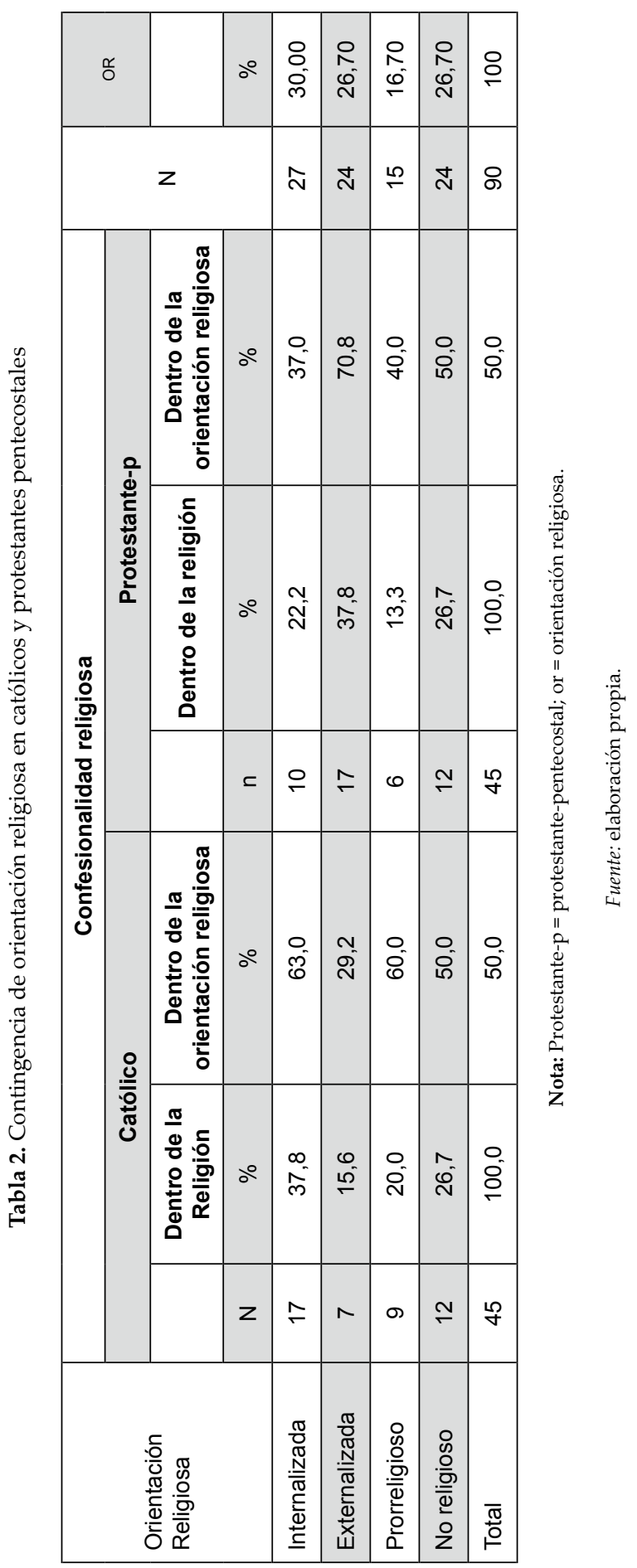


Los resultados de los grupos según su confesionalidad cristiana (católicos y protestantes pentecostales sin entrenamiento previo en solución de problemas sociales) muestran los siguientes resultados. En el grupo de católicos (véase tabla 3), se observan correlaciones negativas entre la dimensión constructiva, la orientación positiva hacia el problema y la resolución racional de problemas y la puntuación de orientación religiosa externalizada, mientras que en el mismo grupo, se observan correlaciones significativas y positivas entre la dimensión disfuncional, la orientación negativa hacia el problema, el estilo descuidado impulsivo y el estilo evitativo y la orientación religiosa externalizada y el locus de control externo.

En el grupo de protestantes pentecostales se encontraron correlaciones significativas y positivas entre la dimensión disfuncional y las puntuaciones del locus de control y orientación religiosa externalizada, y correlaciones significativas y negativas entre la dimensión disfuncional, la orientación negativa al problema y la orientación religiosa internalizada. En la dimensión constructiva se observan correlaciones negativas entre la orientación positiva al problema y el locus de control (interno), y positivas entre la orientación positiva al problema y la orientación religiosa internalizada

En el total de católicos y protestantes, se mantienen las correlaciones positivas entre la dimensión constructiva, la orientación positiva al problema, la resolución racional de problemas y la orientación religiosa internalizada, y correlaciones significativas y negativas con la orientación religiosa externalizada y el locus de control. Puntuaciones altas en el locus de control se relacionan con puntuaciones bajas de la dimensión constructiva.

En el total de católicos y protestantes pentecostales, se mantienen las correlaciones significativas y positivas entre la dimensión disfuncional y la orientación religiosa externalizada y el locus de control, y correlaciones significativas y negativas entre la dimensión disfuncional y la orientación religiosa internalizada.

El análisis multivariado de la varianza (MANOva) con los grupos de católicos y protestantes pentecostales, teniendo en cuenta como variables independientes el locus de control (interno, medio y externo) y a la orientación religiosa (prorreligiosa, no religiosa, intrínseca y extrínseca), y como variables dependientes las puntuaciones de las dimensiones del SPSI-R, la tabla 4 muestra las medias y desviaciones típicas de las puntuaciones de las dimensiones del spsi-R de los grupos de católicos y protestantes pentecostales, de acuerdo con la orientación religiosa y el locus de control. 


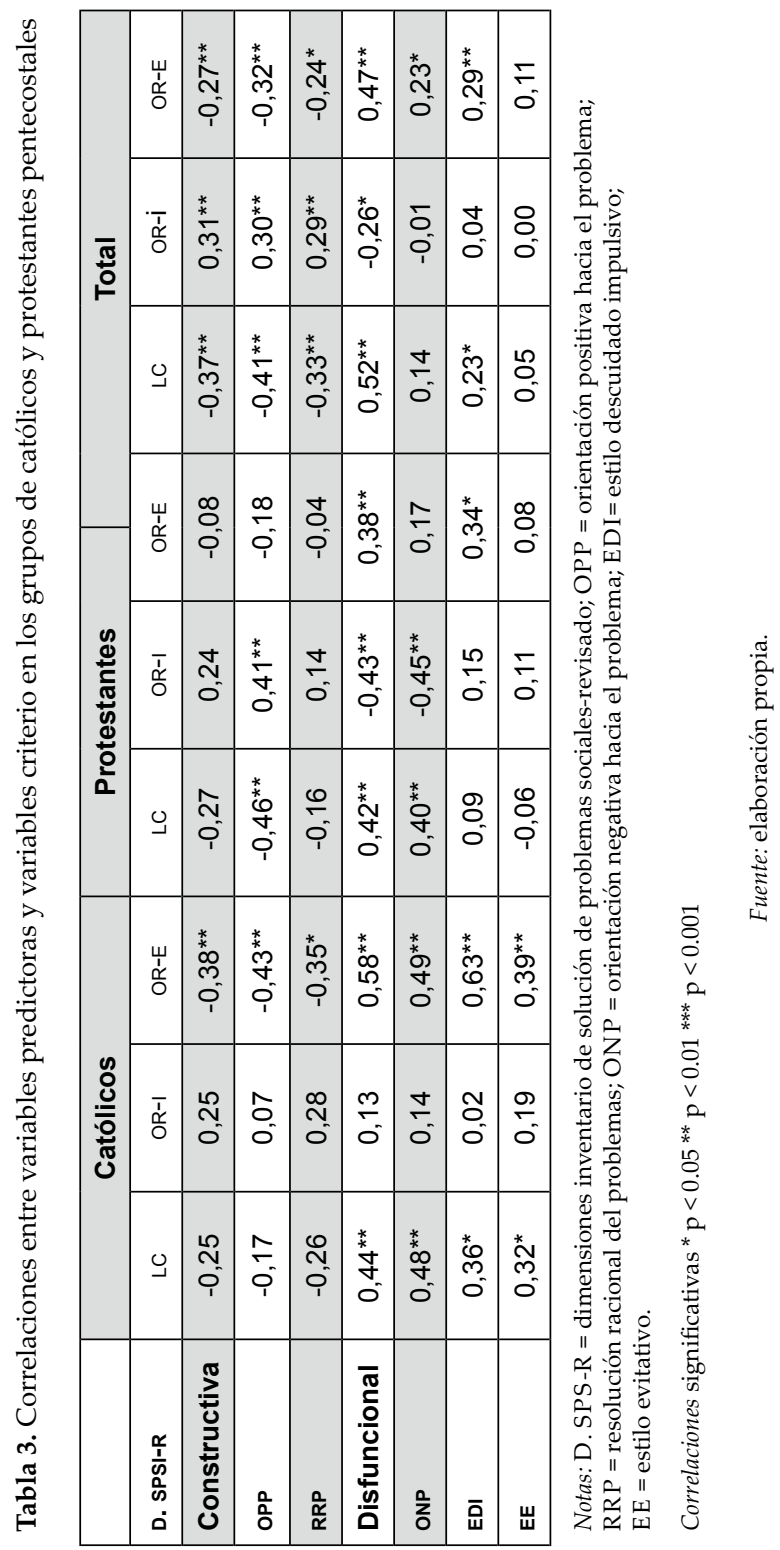




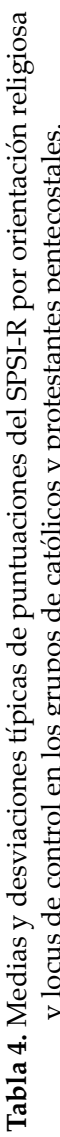

\begin{tabular}{|c|c|c|c|c|c|c|c|c|c|c|c|}
\hline & \multirow{6}{*}{ 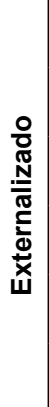 } & \multirow{2}{*}{ 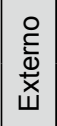 } & 흐 & $\begin{array}{l}0 \\
\infty\end{array}$ & $\stackrel{\vec{m}}{\circ}$ & $\stackrel{\Delta}{\Delta}$ & 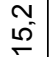 & $\begin{array}{l}0 \\
0^{-}\end{array}$ & $\stackrel{\leftrightarrow}{\sim}$ & 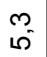 & $\bar{\nabla}$ \\
\hline & & & $1 x$ & $\begin{array}{l}10 \\
0 \\
0\end{array}$ & $\begin{array}{l}m \\
O^{\prime}\end{array}$ & $\begin{array}{c}N \\
o \\
q\end{array}$ & $\begin{array}{l}N \\
\infty \\
\infty \\
m\end{array}$ & \pm & 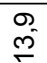 & $\begin{array}{l}m \\
0 \\
0\end{array}$ & $\mathbb{\Xi}_{\mathbb{V}}$ \\
\hline & & 음 & ப & $\overline{\check{m}}$ & 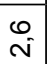 & $\hat{\circ}$ & $\overline{+}$ & $\infty$ & $\stackrel{N}{\infty}$ & $\stackrel{N}{N}$ & : \\
\hline & & $\sum^{0}$ & IX & m & $\stackrel{\infty}{\sim}$ & $\stackrel{10}{\stackrel{N}{N}}$ & $\begin{array}{l}0 \\
\stackrel{0}{0} \\
\text { गे }\end{array}$ & $\begin{array}{l}\stackrel{n}{N} \\
\stackrel{N}{-}\end{array}$ & $\stackrel{1}{\stackrel{0}{\sim}}$ & $\begin{array}{l}10 \\
0 \\
0\end{array}$ & . \\
\hline & & 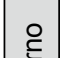 & t & $\check{F}$ & $\stackrel{n}{\sim}$ & $\stackrel{\stackrel{N}{\sim}}{\stackrel{5}{\sim}}$ & $\begin{array}{c}m \\
\stackrel{m}{\sim}\end{array}$ & $\begin{array}{l}N \\
\tilde{N}\end{array}$ & $\stackrel{0}{m}$ & م & \\
\hline & & $\stackrel{\Phi}{\Xi}$ & $1 x$ & $\begin{array}{l}\text { ñ } \\
\text { กิ }\end{array}$ & $\hat{\sigma}$ & $\hat{\widetilde{Y}}$ & $\widehat{\widetilde{m}}$ & $\approx$ & $\stackrel{\infty}{\Gamma}$ & $\widehat{\infty^{\circ}}$ & 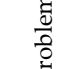 \\
\hline \multirow{18}{*}{ 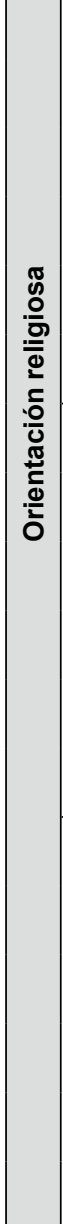 } & \multirow{6}{*}{ 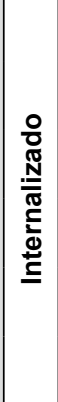 } & 읃 & t & * & * & * & * & $*$ & * & * & \\
\hline & & 齐 & Ix & $\hat{\Lambda}$ & $\stackrel{\bullet}{\sim}$ & $\bar{\epsilon}$ & m్ల & 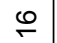 & $\infty$ & $a$ & \\
\hline & & 으 & t艹 & $\begin{array}{l}m \\
m \\
0\end{array}$ & $\hat{m}$ & $\begin{array}{l}0 \\
\stackrel{\sim}{\simeq}\end{array}$ & $\overline{10}$ & $\bar{i}$ & $\lambda$ & $\begin{array}{l}\text { S } \\
\forall\end{array}$ & \\
\hline & & $\sum^{\infty}$ & IX & $\begin{array}{l}\infty \\
\infty \\
0^{\circ}\end{array}$ & $\begin{array}{l}\stackrel{⿱}{\sim} \\
\stackrel{2}{*}\end{array}$ & $\begin{array}{l}\dot{ } \\
\dot{\nabla}\end{array}$ & $\begin{array}{l}0 \\
\stackrel{0}{\sim}\end{array}$ & $\stackrel{m}{F}$ & $\widehat{\infty^{-}}$ & $\stackrel{0}{N}$ & \\
\hline & & $\stackrel{\circ}{\subseteq}$ & t艹 & $\begin{array}{l}10 \\
\tilde{m}\end{array}$ & $\stackrel{+}{\sim}$ & $\hat{\mathrm{N}}$ & $\stackrel{0}{\sim}$ & $\begin{array}{l}0 \\
N \\
N\end{array}$ & $\stackrel{\nabla}{\forall}$ & $\begin{array}{c}\infty \\
\sim \\
\sim\end{array}$ & \\
\hline & & $\stackrel{\oplus}{\Xi}$ & $1 x$ & $\overline{0}$ & \begin{tabular}{l}
$\stackrel{N}{\sim}$ \\
\multirow{F}{*}{}
\end{tabular} & $\begin{array}{l}\text { क } \\
\text { o } \\
\text { in }\end{array}$ & $\begin{array}{l}\infty \\
\stackrel{\infty}{0} \\
-\end{array}$ & $\begin{array}{l}\infty \\
\infty \\
\infty\end{array}$ & $\sigma_{0}^{+}$ & $\begin{array}{l}10 \\
\forall\end{array}$ & \\
\hline & \multirow{6}{*}{ 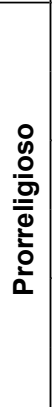 } & $\stackrel{\circ}{\subseteq}$ & t艹 & 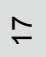 & $\stackrel{10}{N}$ & $\begin{array}{l}10 \\
10 \\
5\end{array}$ & $\begin{array}{l}0 \\
\sigma^{2}\end{array}$ & $\stackrel{a}{N}$ & $\underset{0}{N}$ & $\stackrel{0}{0}$ & \\
\hline & & 这 & 1૪ & $\begin{array}{l}m \\
\text { m } \\
\tilde{L}^{\prime}\end{array}$ & $\begin{array}{l}\text { m } \\
\stackrel{N}{\sim}\end{array}$ & $\stackrel{\mathscr{P}}{\forall}$ & $\hat{\stackrel{2}{0}}$ & $\hat{0}$ & 음 & の & \\
\hline & & 윽 & t & $\begin{array}{l}m \\
\text { ก }\end{array}$ & $\underset{n}{N}$ & $\begin{array}{l}\sigma \\
\sigma \\
\sigma\end{array}$ & $\begin{array}{l}m \\
\stackrel{m}{\sim}\end{array}$ & \begin{tabular}{l|}
0 \\
$\infty$ \\
$\infty$
\end{tabular} & $\hat{\sigma}$ & $\stackrel{N}{N}$ & \\
\hline & & $\sum^{\infty}$ & IX & 요 & $\begin{array}{l}\stackrel{\sim}{\sim} \\
\stackrel{2}{*}\end{array}$ & $\begin{array}{l}0 \\
\dot{\sigma} \\
\dot{\sigma}\end{array}$ & 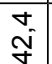 & \begin{tabular}{|l|} 
\\
$\underline{n}$ \\
\end{tabular} & $\begin{array}{l}\infty \\
0^{\circ}\end{array}$ & $\begin{array}{l}\infty \\
\sigma^{\infty}\end{array}$ & \\
\hline & & $\stackrel{\circ}{\varrho}$ & t & $\stackrel{\Delta}{\sim}$ & $m$ & $\underset{10}{N}$ & $\Lambda$ & $\begin{array}{l}\dot{\forall} \\
\dot{m}\end{array}$ & $\stackrel{m}{m}$ & $\hat{\sim}$ & \\
\hline & & $\stackrel{\Phi}{\stackrel{\Phi}{\Xi}}$ & $1 x$ & $\begin{array}{l}m \\
\stackrel{n}{n}\end{array}$ & $\begin{array}{l}\infty \\
\underset{\leftarrow}{+}\end{array}$ & $\begin{array}{l}10 \\
8 \\
8\end{array}$ & \begin{tabular}{l}
$\infty$ \\
$0^{\infty}$ \\
\hdashline
\end{tabular} & $\begin{array}{l}\mathcal{N} \\
\dot{f}\end{array}$ & $\stackrel{N}{m}$ & $\begin{array}{l}\infty \\
\tilde{m}\end{array}$ & \\
\hline & \multirow{6}{*}{ 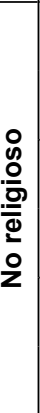 } & $\stackrel{\circ}{\underline{t}}$ & t艹 & $\stackrel{\check{\sigma}}{\check{r}}$ & $\begin{array}{l}\infty \\
m\end{array}$ & $\stackrel{m}{m}$ & $\hat{\bar{N}}$ & $\infty$ & $\infty$ & $\stackrel{N}{0}$ & \\
\hline & & 离 & $1 x$ & 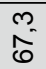 & \begin{tabular}{l}
$m$ \\
\multirow{F}{*}{}
\end{tabular} & గొ & $\begin{array}{l} \\
\text { N. } \\
\text { m }\end{array}$ & $\stackrel{\varphi}{\circ}$ & $\begin{array}{l}\infty \\
0^{-}\end{array}$ & $\mp$ & \\
\hline & & 응 & t艹 & $\underset{\sigma}{\mathscr{V}}$ & $\begin{array}{l}\infty \\
\forall\end{array}$ & 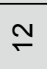 & $\begin{array}{l}0 \\
\stackrel{-}{\circ}\end{array}$ & $\underset{⿱ 宀}{*}$ & $\bar{\nabla}$ & $\nabla$ & \\
\hline & & $\sum^{\infty}$ & Ix & ठี & $\stackrel{m}{\square}$ & in & $\stackrel{\hat{\sigma}}{\sigma}$ & $\widehat{\sigma^{-}}$ & $\bar{i}$ & $\stackrel{0}{\sigma 0}$ & \\
\hline & & ๑ & t & $\bar{\sigma}$ & $\begin{array}{l}\infty \\
\boldsymbol{n}^{-}\end{array}$ & $\begin{array}{l}\tilde{L} \\
\stackrel{2}{\Gamma}\end{array}$ & $\hat{N}$ & $\begin{array}{l}\infty \\
\boldsymbol{m}^{-}\end{array}$ & $\stackrel{\infty}{\infty}$ & $\stackrel{\sim}{m}$ & \\
\hline & & $\stackrel{\oplus}{\Xi}$ & $1 x$ & $\mathscr{C}$ & $\begin{array}{l}\infty \\
\stackrel{-}{\sim}\end{array}$ & กิ & $\begin{array}{l}m \\
\stackrel{0}{0} \\
-\end{array}$ & $\begin{array}{l}0 \\
0 \\
10\end{array}$ & 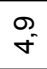 & $\begin{array}{l}\infty \\
\omega^{-}\end{array}$ & $\infty$ \\
\hline & & & 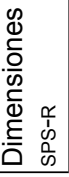 & 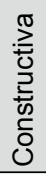 & $\begin{array}{l}0 \\
0 \\
0\end{array}$ & 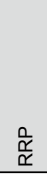 & 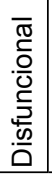 & $\begin{array}{l}0 \\
z \\
0\end{array}$ & 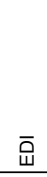 & 岀 & \\
\hline
\end{tabular}


En los grupos de católicos y protestantes-pentecostales se encontraron efectos principales de locus de control y orientación religiosa, y efectos de la interacción entre ambas variables (LC+OR) respecto a las puntuaciones de las dimensiones del SPSI-R. La tabla 5 muestra los valores $F$ resultantes de MANOvA.

Tabla 5. Valores $\boldsymbol{F}$ del análisis multivariado de la varianza para las puntuaciones del spsi-R, de acuerdo con la orientación religiosa y el locus de control

\begin{tabular}{|l|c|c|c|}
\hline Dimensiones SPSI-R & $\begin{array}{c}\text { Locus de control } \\
\text { (LC) }\end{array}$ & $\begin{array}{c}\text { Orientación } \\
\text { Religiosa (OR) }\end{array}$ & LC + o R \\
\hline Constructiva & $3,91^{*}$ & $6,92^{* * *}$ & 1,21 \\
\hline OPP & 1,69 & $5,39^{* * *}$ & 0,30 \\
\hline RRP & $3,86^{*}$ & $5,85^{\star * *}$ & 1,31 \\
\hline Disfuncional & $4,22^{*}$ & $4,12^{* *}$ & $2,48^{*}$ \\
\hline ONP & $5,14^{* *}$ & $5,14^{* *}$ & $3,42^{* *}$ \\
\hline EDI & 2,11 & $5,35^{* *}$ & $2,75^{*}$ \\
\hline EE & $3,35^{*}$ & 0,69 & 0,78 \\
\hline
\end{tabular}

Nota: dimensiones SPS-R = dimensiones del inventario de solución de problemas sociales revisado; $\mathrm{OPP}=$ orientación positiva hacia el problema; $\mathrm{RRP}=$ resolución racional del problema;

$\mathrm{ONP}$ = orientación negativa hacia el problema; $\mathrm{EDI}=$ estilo descuidado impulsivo; $\mathrm{EE}=$ estilo evitativo.

Correlaciones significativas ${ }^{*} \mathrm{p}<0.05^{* *} \mathrm{p}<0.01^{* * *} \mathrm{p}<0.001$.

Fuente: elaboración propia.

En primer lugar, se observa que el locus de control influye sobre las puntuaciones de la dimensión constructiva, la resolución racional de problemas la dimensión disfuncional, la orientación negativa hacia el problema y el estilo evitativo de solución de problemas sociales. Adicionalmente, la orientación religiosa influye de forma significativa en las puntuaciones de todas las dimensiones del inventario, excepto en el estilo evitativo. La interacción entre orientación religiosa y locus de control influyó sobre las puntuaciones de las dimensiones disfuncional, orientación negativa hacia el problema y estilo descuidado impulsivo. Las pruebas Post-Hoc y las medias marginales (tabla 6) ilustran de forma más específica las diferencias de los grupos de orientación religiosa y locus de control respecto a las puntuaciones de las dimensiones del SPSI-R.

En la tabla 6 se pueden apreciar las diferencias significativas en las puntuaciones de las dimensiones del SPSI-R del grupo de orientación religiosa externalizada respecto a los demás grupos de orientación religiosa. Los participantes con orientación religiosa externalizada tienen menores puntuaciones en la dimensión constructiva, la orientación positiva hacia el problema y la resolución racional de problemas que los participantes con orientación religiosa internalizada, no religiosos y prorreligiosos. Al mismo tiempo, este grupo de orientación religiosa externalizada presenta puntuaciones más altas en la dimensión disfuncional, la orientación negativa hacia el problema, el estilo descuidado impulsivo y el estilo evitativo. 


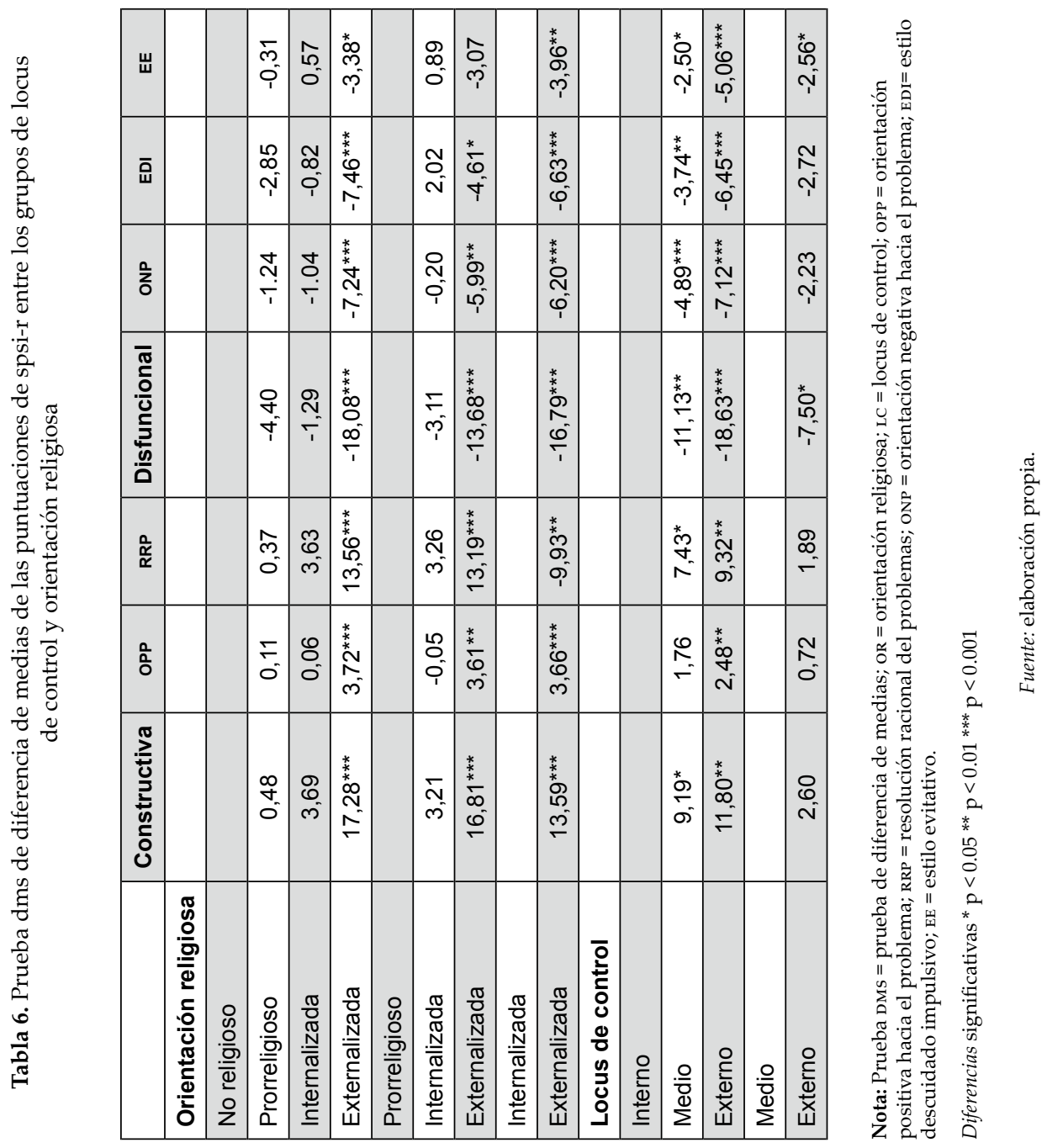


Por otra parte, se encontró que las diferencias en función del locus de control se presentan en todas las dimensiones del SPSI-R, entre el grupo de locus de control interno y externo. El grupo con locus de control interno presenta puntuaciones más altas que el locus de control externo en la dimensión constructiva, la orientación positiva hacia el problema y la resolución racional de problemas, y puntuaciones más bajas que el mismo grupo en la dimensión disfuncional, la orientación negativa hacia el problema, el estilo descuidado impulsivo y el estilo evitativo.

La comparación entre los grupos de locus de control interno y medio tiene las mismas características de la comparación anterior, excepto por una ausencia de diferencias significativas en la orientación positiva hacia el problema. Por su parte, el grupo con locus de control externo presenta puntuaciones más altas en el estilo evitativo que el grupo con locus de control medio.

Finalmente, se realizó una comparación en las dimensiones de solución de problemas del SPSRI-R entre católicos y protestantes pentecostales del grupo control, mediante la prueba $t$ para muestras independientes. Los resultados indican que los participantes católicos tienen promedios significativamente más altos en las puntuaciones de la dimensión constructiva, la orientación positiva hacia el problema y la resolución racional de problemas, mientras que los participantes protestantes pentecostales mostraron puntuaciones significativamente más altas en la dimensión disfuncional, orientación negativa hacia el problema, estilo descuidado impulsivo y estilo evitativo (véase tabla 7).

Tabla 7. Medias y desviaciones típicas y puntuaciones $t$ para las comparaciones de puntuaciones del sPSI-R correspondientes a católicos y protestantes pentecostales

\begin{tabular}{|l|c|c|c|c|c|}
\hline \multirow{2}{*}{ Dimensiones SPS-R } & \multicolumn{2}{|c|}{ Católicos } & \multicolumn{2}{c|}{ Protestantes-p } & \multirow{2}{*}{$\boldsymbol{t}$} \\
\cline { 2 - 5 } & & DT & & DT & \\
\hline Constructiva & 65,2 & 16,8 & 53,8 & 12,6 & $3,65^{\text {*** }}$ \\
\hline OPP & 13,6 & 3,8 & 11,2 & 3,6 & $3,17^{\text {** }}$ \\
\hline RRP & 51,6 & 13,8 & 42,6 & 10,7 & $3,44^{\text {***}}$ \\
\hline Disfuncional & 21,2 & 14,3 & 33,5 & 17,4 & $-3,67^{\text {***}}$ \\
\hline ONP & 8,1 & 5,8 & 12,4 & 7,4 & $-3,05^{\text {** }}$ \\
\hline EDI & 7 & 5,8 & 12,1 & 6,5 & $-3,95^{\text {** }}$ \\
\hline EE & 6,1 & 4,6 & 9 & 5,2 & $-2.86^{* *}$ \\
\hline
\end{tabular}

Nota: Protestante- $\mathrm{p}=$ protestante-pentecostal; = medias; $\mathrm{DT}=$ desviación típica; dimensiones sPS- $\mathrm{R}=$ dimensiones del inventario de solución de problemas sociales revisado; oPP = orientación positiva hacia el problema; $\mathrm{RRP}$ = resolución racional del problemas; $\mathrm{ONP}$ = orientación negativa hacia el problema; $\mathrm{EDI}=$ estilo descuidado impulsivo; $\mathrm{EE}=$ estilo evitativo.

Diferencias significativas ${ }^{*} \mathrm{p}<0.05^{* *} \mathrm{p}<0.01^{* * *} \mathrm{p}<0.001$. 


\section{Discusión}

En el total de las correlaciones entre variables predictoras y variables criterio, se observa que tanto en católicos como en protestantes pentecostales se mantienen correlaciones positivas entre las escalas de la dimensión constructiva y la orientación religiosa internalizada, y correlaciones negativas con la orientación religiosa externalizada. Por su parte, en la dimensión disfuncional se mantienen correlaciones negativas con la orientación religiosa internalizada y correlaciones positivas con la orientación religiosa externalizada.

Dentro de los grupos sin exposición a entrenamiento en solución de problemas, se comprueba la hipótesis 1, según la cual las personas con mayores puntuaciones en la dimensión constructiva y menores en la dimensión disfuncional tenderán a la orientación religiosa internalizada, independiente de su confesionalidad cristiana.

A partir de las correlaciones entre variables predictoras y variables criterio en los grupos de católicos y protestantes pentecostales (tabla 3), se acepta la hipótesis 2: las personas con orientación religiosa internalizada, sin exposición a entrenamiento en solución de problemas, presentan correlaciones significativas y positivas con la dimensión constructiva, la orientación positiva al problema y la resolución racional de problemas; y correlaciones significativas y negativas con la puntuación de la orientación religiosa externalizada.

Por su parte, las personas con orientación religiosa externalizada, sin exposición a entrenamiento en solución de problemas, presentan correlaciones significativas y negativas entre las escalas de la dimensión disfuncional y la orientación religiosa internalizada, y correlaciones positivas entre la dimensión disfuncional, la orientación negativa al problema y el estilo descuidado impulsivo y la orientación externalizada.

Se comprobó que las personas con orientación religiosa internalizada, sin exposición a entrenamiento en solución de problemas, tienden a obtener mayores puntuaciones en la dimensión constructiva, y menores en la dimensión disfuncional, que las personas con orientación religiosa externalizada.

$\mathrm{Al}$ abordar la hipótesis 3, se encuentran en los grupos de católicos y protestantes pentecostales efectos principales del locus de control y orientación religiosa, y efectos de la interacción entre ambas variables (LC+OR) respecto a las puntuaciones de las dimensiones del SPSI-R. Se verifica esta hipótesis cuando las personas sin entrenamiento en solución de problemas sociales, con orientación religiosa internalizada y locus de control interno tienden a obtener altas puntuaciones en la dimensión constructiva, mientras que las personas con orientación religiosa externalizada y locus de control externo tienden a puntuar más en la dimensión disfuncional. 


\section{Conclusiones}

1. En los grupos de católicos y protestantes pentecostales, las personas con orientación religiosa internalizada obtienen altas puntuaciones en la dimensión constructiva, y bajas puntuaciones en la dimensión disfuncional, mientras que las personas con orientación religiosa externalizada tienen bajas puntuaciones en la dimensión constructiva y altas puntuaciones en la dimensión disfuncional.

2. En los grupos de católicos y protestantes pentecostales, las personas con orientación religiosa internalizada y locus de control interno obtienen altas puntuaciones en la dimensión constructiva y bajas puntuaciones en la dimensión disfuncional, mientras que las personas con orientación religiosa externalizada y locus de control externo tienen altas puntuaciones en la dimensión disfuncional y bajas puntuaciones en la dimensión constructiva.

3. La confesionalidad religiosa influye sobre las puntuaciones de la dimensión constructiva y disfuncional del grupo de control en solución de problemas sociales.

4. Teniendo en cuenta que la mayor parte de los colombianos pertenecen a la tradición cristiana, desde las confesionalidades católica y protestante pentecostal, es un hecho que la mayoría de consultantes en el campo de la psicología en Colombia ven la capacidad de intervención - constructiva o disfuncional- de los factores religiosos en la solución de problemas.

\section{Referencias}

Allport, G. W. y Ross, J. M. (1967). Personal religious orientation and prejudice. Journal of Personality and Social Psychology, 5(4), 432-443.

Arana, J. (2007). Temas centrales del diálogo ciencia-fe en la actualidad. Scripta Theologica, 39(2), 479-494.

Ardila, R. (2005). La ciencia y los científicos. Una perspectiva psicológica. Medellín: Universidad de Antioquia.

Artigas, M. (2011). Ciencia, razón y fe (2. ${ }^{a}$ ed.). Navarra: Eunsa.

Ávila, A. (2003). Para conocer la psicología de la religión. Estella: Verbo Divino.

Ávila, S. R. (2009). Psicología y religión: un enfoque conductual. México: Manual Moderno. 
Beltrán, W. M. (2011). Descripción cuantitativa de la pluralización religiosa en Colombia. Universitas Humanística, (73), 201-237.

Boff, L. (2001). Gracia y experiencia humana. Madrid: Trotta.

Comisión Teológica Internacional (2012). Theology today: Perspectives, principles and criteria. Madrid: Biblioteca de Autores Cristianos. [Traducción al español de José Luis Mogica].

Consejo Episcopal Latinoamericano y del Caribe. (2007). La educación católica. En v Conferencia General del Episcopado Latinoamericano y del Caribe. Bogotá: Consejo Episcopal Latinoamericano y del Caribe.

Croatto, J. S. (2002). Experiencia de lo sagrado y tradiciones religiosas. Estudio de fenomenología de la religión. Estella: Verbo Divino.

D'Zurilla, T. J. (1986). Problem-solving therapy: A social competence approach to clinical intervention. Nueva York: Springer.

D'Zurilla, T. J. (1988). Problem-solving therapies. En K. S. Dobson (Ed.), Handbook of cognitive behavioral therapies (pp. 85-135). Nueva York: Guilford.

D'Zurilla, T. J. y Goldfried, M. (1971). Problem solving and behavior modification. Journal of Abnormal Psychiatry, 78, 107-126.

D'Zurilla, T. J., Nezu, A. M. y Maydeu Olivares, A. (1999). Manual for the social problem-solving inventory-revised. North Tonawanda, NY: Multi-Health Systems.

D’Zurilla, T. J., Nezu, A. M. y Maydeu Olivares, A. (2002). Social problem-solving inventory-revised (SPSI-R): Technical manual. North Tonawanda, NY: Multi Health Systems.

D' Zurilla, J. T., Nezu, A. M. y Maydeu Olivares, A. (2004). Social problem solving: Theory and assessment. En E. C. Chang, J. T. D'Zurilla, y L. J. Sanna (eds.), Social problem solving. Theory, research, and training (pp. 11-27). Washington, D. C.: American Psychological Association.

De la Torre. T. M., Morera F. O., y Wood, M. J. (2010). Measuring social problem solving using the Spanish version for Hispanics of the social problem solving inventory-revised. Cultural Diversity and Ethnic Minority Psychology, 16(4), 501-506.

Dei, V. (1965). Sobre la Divina Revelación. En Documentos del Vaticano II: constituciones, decretos, declaraciones. Madrid: Biblioteca de Autores Cristianos.

Donahue M. J. (1985). Intrinsic and extrinsic religiousness: Review and meta analysis. Journal of Personality and Social Psychology, 48(2), 400-419. 
Emmons, R. A., y Paloutzian, R. F. (2003). The psychology of religion. Annual Review of Psychology, 54, 377-402.

Evans, J. H. (2011). Epistemological and moral conflict between religion and science. Journal for the Scientific Study of Religion, 50(4), 707-727.

Fliegel, K. G., y Schulte, S. (1989). Métodos standard de la terapia del comportamiento. Bogotá: Unión Gráfica.

García, J. L. (2008). Aproximación epistemológica al concepto de ciencia: una propuesta básica a partir de Kuhn, Popper, Lakatos y Feyerabend. Andamios, 4(8), 185-212.

García, L. F. (2012). Protestantes, evangélicos y pentecostales: aclaraciones conceptuales preliminares en un campo de investigación social. Folios, 36, 171-187.

Ioannes, P. PP. II. (1998). Fides et ratio. A los obispos de la Iglesia católica sobre las relaciones entre fe y razón. Ciudad del Vaticano: Editrice Vaticana.

James, W. (1902). The varieties of religious experience: A study in human nature. Edinburgh: Longmans, Green, and Co.

Jaume, L., Simkin, H. y Eychezahar, E. (2013). Religious as quest and its relationship with intrinsic and extrinsic orientation. International Journal of Psychological Research, 6(2), 71-78.

Krauss, S. W. y Hood, R. W. (2013). New approach to religious orientation: The Commitment-reflectivity circumplex. Amsterdam: Rodopi B. V.

McMinn, M. R., Hathaway, W. L., Woods, S. W. y Snow, K. N. (2009). What American Psychological Association leaders have to say about psychology of religion and spirituality. Psychology of Religion and Spirituality, 1(1), 3-13.

Maydeu-Olivares, A., y D'Zurilla, J. T. (1995). A factor analysis of the social problem-solving inventory using polychoric correlations. European Journal of Psychological Assessment, 1(2), 98-107.

Merino, C. (2012). Forma breve del spsi-R: análisis preliminar de su validez interna y confiabilidad. Terapia psicológica. Sociedad Chilena de Psicología Clínica, 30(2), 85-90.

Ministerio del Interior y Justicia. (2014). Asuntos religiosos. Registro Público de Entidades Religiosas. Recuperado de http://www.mininterior.gov.co/mision/ asuntos-religiosos/registro-publico-de-entidades-religiosas

Muñoz, A. (2004). Cuestiones epistemológicas relativas al estudio psicológico de la vivencia religiosa. PSYKHE, 13(1), 131-140. 
Nezu, A. M. y Nezu, C. M. (1989). Clinical decision making in behavior therapy: A problem-solving perspective. Champaign, Ill.: Research Press.

Nezu, A. M., Nezu, C. M. (1991). Entrenamiento en solución de problemas. En V. Caballo (dir.), Manual de técnicas de terapia y modificación de conducta (pp. 527-553). Madrid: Siglo xxI.

Nezu, A. M., Nezu, C. M. y Lombardo, E. (2004). Formulación de casos y diseño de tratamientos cognitivo-conductuales. Un enfoque basado en problemas. México: El Manual Moderno.

Nezu, A. M., Nezu, C. M. y Perri, M. G. (1989). Problem-solving therapy for depression: Theory, research, and clinical guidelines. Nueva York: Wiley.

Newell, A. y Simon, H. (1972). Human problem solving. Englewood Cliffs, NJ: Prentice-Hall.

Pérez, A. A., Guerrero, F. y López, L. W. (2002). Siete conductismos contemporáneos: una síntesis verbal y gráfica. International Journal of Psychology and Psychological Therapy, 2(1), 103-113.

Patrick, J. W. (1979). Personal faith and the fear of death among divergent religious populations. Journal for the Scientific Study of Religion, IS, 298-305.

Piaget. J. (1978). What is psychology? American Psychologist, 33(7), 648-652.

Ralph, L. P. (2013). A short history of the psychology of religion and spirituality: Providing growth and meaning for division 36. Psychology of Religion and Spirituality, 5(1), 1-4.

Ralph W. y Hood Jr. (1971). A Comparison of the Allport and Feagin scoring procedures for intrinsic/extrinsic religious orientation. Journal for the Scientific Study of Religion, 10(4), 370-374.

Romero, M. J. (2011). De las ciencias a la teología. Ensayos interdisciplinares. Estella: Verbo Divino.

Rotter, J. B. (1966). Generalized expectancies for internal versus external control of reinforcement. Psychological Monographs: General and Applied, 80(60). Recuperado de http://www.soc.iastate.edu/sapp/soc512Rotter.pdf

Sánchez H. Y. (2007). La solución de problemas como un campo de concurrencia de distintas teorías en psicología. Revista Colombiana de Psicología, (16), 147-162.

Scioli, A., Ricci, M., Nyugen, T. y Scioli, E. (2011). Hope: Its nature and measurement. Psychology of Religion and Spirituality, 3(2), 78-97. 
Skinner B. F. (1953). Science and Human Behavior. The free Press. Nueva York: Macmillan.

Skinner B. F. (1966). What is the experimental analysis of behavior. Journal of the Experimental Analysis of Behavior, 9(3), 213-218.

Skinner B. F. (1974). Sobre el conductismo. Barcelona: Martínez Roca.

Skinner B. F. (1986). What is wrong with daily life in the western world? American Psychologist, 41(5), 568-574.

Starbuck, D. E. (1901). The psychology of religion: An empirical study of the growth of religious consciousness (3.a ed.). The Walter Scott Publishing Co. Stark, R. y Glock, C. Y. (1970). American piety: The nature of religious commitment. Berkeley: University of California Press.

Syeda, T. Z. y Rabia, M. (2011). Religious orientation and locus of control among madrassa and university students. Pakistan Journal of Psychology, 42(1), 67-85.

Torres, Q. A. (2008). Repensar la revelación divina en la realización humana. Madrid: Trotta.

Vasco, U. C., Escobedo, D. H., León, P. T. y Negret, P. J. (1995). La teoría general de procesos y sistemas. Una propuesta semiológica, ontológica y gnoseológica para la ciencia, la educación y el desarrollo. Bogotá: Imprenta Nacional.

Visdómine Lozano, J. C. y Luciano, C. (2006). Locus de control y autorregulación conductual: revisiones conceptual y experimental. International Journal of Clinical and Health Psychology, 6(3), 729-751.

Walker, D. F., Worthington, E. L., Gartner, A. L., Gorsuch, R. L., y Rhodes, H. E. (2011). Religious commitment and expectations about psychotherapy among Christian clients. Psychology of Religion and Spirituality, 3(2), 98-114.

Watson, J. B. (1913). Psychology as the behaviorist views it. Psychological Review, 20(2), 158-177. 